\title{
Image-Based Measurement of Alveoli Volume Expansion in an Animal Model of a Diseased Lung
}

\author{
C. E. Hann ${ }^{1}$, D. Hewett ${ }^{1}$, J. G. Chase ${ }^{1}$, T. Rabczuk ${ }^{1}$, A. Sundaresan ${ }^{1}$, X. Chen ${ }^{1}$, W. Wang ${ }^{1}$ and G. M. Shaw ${ }^{2}$ \\ ${ }^{1}$ Department of Mechanical Engineering, University of Canterbury, Christchurch, New Zealand \\ ${ }^{2}$ Department of Intensive Care, Christchurch Hospital, Christchurch, New Zealand \\ Email: C.E.Hann@canterbury.ac.nz
}

\begin{abstract}
Currently, there does not exist reliable MV treatment or protocols in critical care to treat acute respiratory diseases, and thus no proven way to optimise care to minimise the mortality, length of stay or cost. The overall approach of this research is to improve protocols by using appropriate computer models that take into account the essential lung mechanics. The aim of this research is to create an automated algorithm for tracking the boundary of individual or groups of alveoli, and to convert this into a pressure volume curve for three different types of alveoli. A technique called in vivo microscopy has been developed by Schiller et al which visualizes the inflation and deflation of individual alveoli in a surfactant deactivation model of lung injury in pigs. Three different types of alveoli were tracked using data from Schiller et al, type I, II and III. These types correspond to healthy alveoli, non-collapsing but partially diseased alveoli, and fully collapsing diseased alveoli respectively. The boundaries of all the alveoli that were tracked, compared well visually to the movies. Pressure versus Area curves were derived for both inflation and deflation, they captured the expected physiological behaviour, and were qualitatively similar to the quasi-static pressure area curves derived in Schiller et al, Quantitative differences are due to the dynamic effects of ventilation which were not investigated in Schiller et al.
\end{abstract}

\section{INTRODUCTION}

A significant number of patients admitted to the ICU require mechanical ventilation due to acute respiratory failure, such as Acute Respiratory Distress Syndrome (ARDS) [1] that develops along with their other reasons for admission. The mortality rate of ARDS ranges from $30-70 \%$ and is treated by supporting the lungs using Mechanical Ventilation (MV). MV therapy also adds $33 \%$ to the daily cost per patient and is a marker for almost doubling the length of a patients stay. There are no specific treatments or protocols for using MV to treat acute respiratory diseases, and thus no proven way to optimise care to minimise the mortality, length of stay or cost.

This research focuses on the fundamental dynamics of the ARDS lung by creating real-time patient specific models [2]. These models thus directly represent the physiological status of a given patient. From this computer aided physiological picture, clinical diagnosis and patient specific treatment can be applied.

The stress and strain in the lung are the primary determinants of ventilator-induced lung injury. Therefore, a patient-specific measurement of stress for given ventilator settings is crucial to improving the current protocols in critical care. A recent study [3], determined the stress and strain in the lung as a result of ventilator therapy for a diverse range of patients with different degrees of lung injury. The study used trans-pulmonary pressure (airway pressure minus pleural pressure) as the clinical equivalent of stress, and the ratio of volume change to the functional residual capacity (FRC), as the clinical equivalent of strain. The results in [3], showed that stress to strain relationship was very similar for both acutely injured and healthy lungs, but the degree of stress or strain for a given ventilator settings, varied significantly over all patients. This study motivates the use of animal models to derive a stress threshold that is considered damaging to the lung, which could be potentially used as a first attempt for minimizing lung damage on humans.

However, the stress strain relationship in [3] defines the global behaviour and doesn't take into account the effect on the well known different types of alveoli. This research therefore seeks to characterize the stress strain relationship in three types of alveoli corresponding to healthy alveoli, noncollapsing but partially diseased alveoli, and fully collapsing diseased alveoli. The current modeling approach [2] can account for and identify the different distributions based on pressure volume data for various positive end-expiratory pressure (PEEP) settings. Therefore, once the stress strains laws are known for each alveoli type the contribution to the overall stress in the lung can be computed.

A method for visualizing the inflation and deflation of individual alveoli under various forms of mechanical ventilation has been developed by Schiller et al [4]. The technique is called in vivo microscopy and has been tested on a surfactant deactivation model of lung injury in pigs. The approach produces a number of images for each ventilation cycle and a portion of the data from this experiment forms the basis for the research in this paper. However, manually processing many images from a number of trials, which is required to accurately derive stress strain laws, is very time consuming, prone to error and not practical. The aim in this paper is to create an automated algorithm for tracking the boundary of individual or groups of alveoli, and to convert this into a pressure area curve for three different types of alveoli. Schiller et al [4] did not derive pressure area curves in the dynamic ventilation case, but manually extracted alveoli in images of a quasi-static pressure area curve. Here small 
increments of tidal volume are given so that the pressure reaches a steady state at each step. However, during normal ventilation the steady state pressure is never reached. Thus, the results of Schiller et al [4] do not necessarily reflect the dynamic state of the alveoli during ventilation. Hence the approach in this paper is to develop a general method that can handle this more difficult case of dynamic alveoli tracking.

\section{METHODOLOGY}

\section{A. Experimental setup and image acquisition}

The experimental data comes from anesthetized pigs weighing $15-20 \mathrm{~kg}$, with a surfactant deactivation model for lung injury. Microscopic images were taken at high magnification (100x) with a video camera (CCD SSC-S20, SONY, Tokyo, Japn) and recorded with a video recorder (SVO-9500 MD, SONY). For more detailed information on the protocols and overall method of in vivo microscopy see [4]. The dynamically ventilated alveoli data from [4] was available for the research in this paper. The data was in movie format (MPEG), which was converted to a sequence of .jpg files. The frame rate was 40 images/s which gave typically 35-50 images for each ventilation cycle, depending on the alveoli type. Figures 1 and 2 show an example of the images that result from the method of [4]. Figure 1 is the alveoli after inflation, and Figure 2 is the alveoli after deflation.

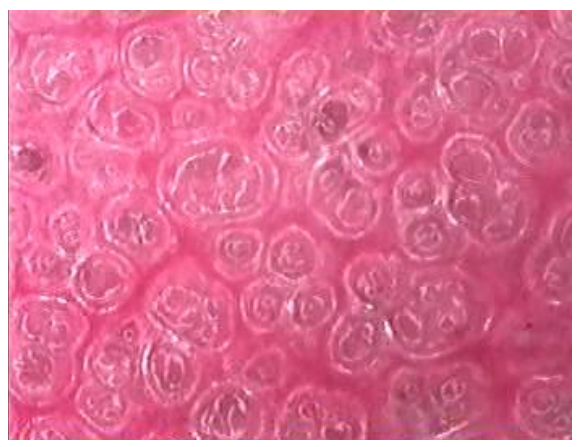

Figure 1. An example of a microscopic image at the end of inflation

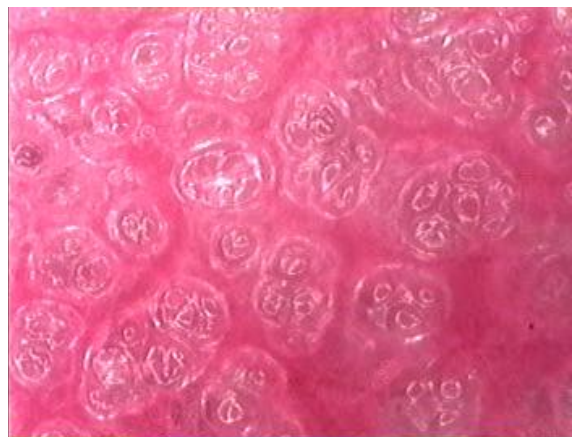

Figure 2. An example of a microscopic image at the end of deflation

\section{B. Alveoli tracking - overall approach and initialization}

The goal is to track three alveoli types 1, 2 and 3 based on the video images from [4]. To ensure consistency, 5 ventilator cycles were tracked for each alveoli type. Taking into account different alveoli or groups of alveoli the number of .jpg images was in the thousands, which is not feasible to analyze manually. Therefore an automated alveoli tracking method is required.

The .jpg files corresponding to the video images from [4], are first read into Matlab for image processing and analysis. There are many sophisticated algorithms for general motion tracking for example the method of snakes [5]. However, to avoid computational issues and significant tuning/outlier rejection, a simpler method specific to the alveoli tracking application was developed. Furthermore, gradient based methods like Canny [6,7] for edge detection were also not used due to the large number of potential gradient changes present due to both the natural features and image noise. Although there exist methods for reducing noise in these methods, they typically require advanced filtering methods for accurate results e.g. [8]. With the general Canny edge detector $[6,7]$ it was found that an excessive number of edges in the alveoli images were found which made it difficult to process the data consistently and accurately. Instead a gradient free method based on colour thresholding was used, which utilizes the known cyclic behaviour of the ventilator.

The approach is to first automatically locate, the time when the alveoli were at their maximum volume in the first ventilator cycle. This time can be easily found by the peak of the airway pressure curve from the ventilator. The image corresponding to the maximum alveoli volume is then presented to a user, and a boundary is manually drawn around the individual or group of alveoli that is required to be tracked.

The boundary ensures that the algorithm will not latch on to surrounding alveoli even though they are sometimes very close. The drawing of the boundary is the only intervention required in the algorithm, but has the added advantage of allowing the freedom of choosing which alveoli to track. Figure 3 shows an example of this initialization, where the whole region of interest is blotted out in black.

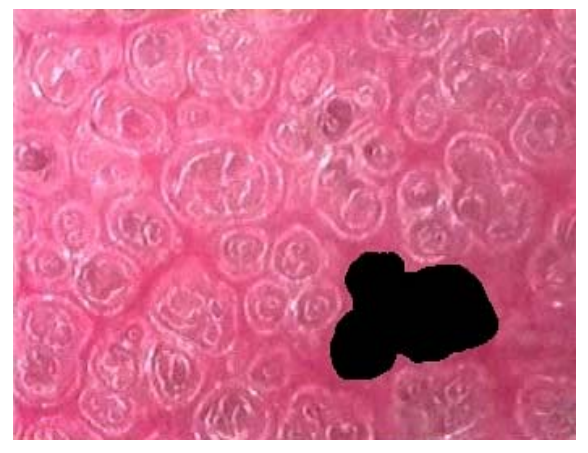

Figure 3. Starting image for an automatic tracking algorithm. The region of interest is filled in black. 
The alveoli in Figure 3 were type 3, so they could collapse at the end of deflation. In some cases, at the end of collapse, alveoli disappeared off the image. Therefore, in these cases a group of alveoli are tracked rather than an individual alveoli. This approach can then give an average response of an alveoli which would be more robust to image noise and uncertainty. A similar method is used on type 2 alveoli for comparison.

The final output of the algorithm is an area versus time graph for the group of alveoli from the beginning of inflation to the end of deflation. The area versus time graph is calculated from the enclosed area of each extracted contour surrounding the group of alveoli for the movie image sequence. In the first half of the cycle, the contour moves from the beginning of inflation (minimum area or collapsed state of alveoli) to the peak inflation frame of Figure 3 (maximum area). In the second half the tracked contour moves from the peak inflation frame back to the alveoli collapsed state. At all stages of the cycle, it is assumed that the group of alveoli lie within the black region shown in Figure 3. This assumption is valid as the alveoli motion is at steady state and is periodic with a fixed amplitude. The algorithm is repeated for as many cycles as required.

\section{Alveoli tracking - contour extraction and area calculation}

The area of the first frame in Figure 3 is defined to be the black region and is measured by the number of pixels.

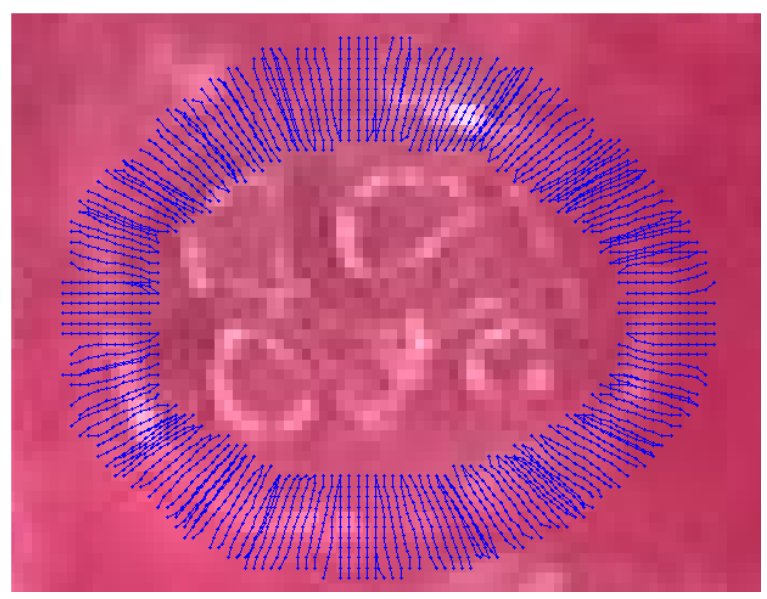

Figure 4. Example of the algorithm looking for edges surrounding a type 3 alveolus group.

The contour of this region serves as a starting point for finding the edge of the alveolus group in the next frame. This new edge is extracted by first looking radially inwards from each point on the prior contour, since this is the direction the collapsing alveoli must travel. Figure 4 shows an example of the algorithm looking for edges in a neighbourhood of the alveolus group. The number of pixels that are investigated along the radial line is chosen to be 11 based on experiment.

The blue lines in Figure 4 depict the path over which the colour intensities are analyzed to find an edge. Starting from the position of the previous edge point, the sum of the red and green channels is investigated along the radial line pointing inwards. If a point is reached where the sum changes by a given threshold from the initial starting point, this point is defined as the new edge point. This approach and threshold was found from experiment, and gave reasonable results over all the images investigated. Note that since a combination of the colour channels is used to characterize an edge it is less sensitive to noise since no gradients are required. The end result is points both on the edge of the alveolus and inside it as shown in Figure 5.

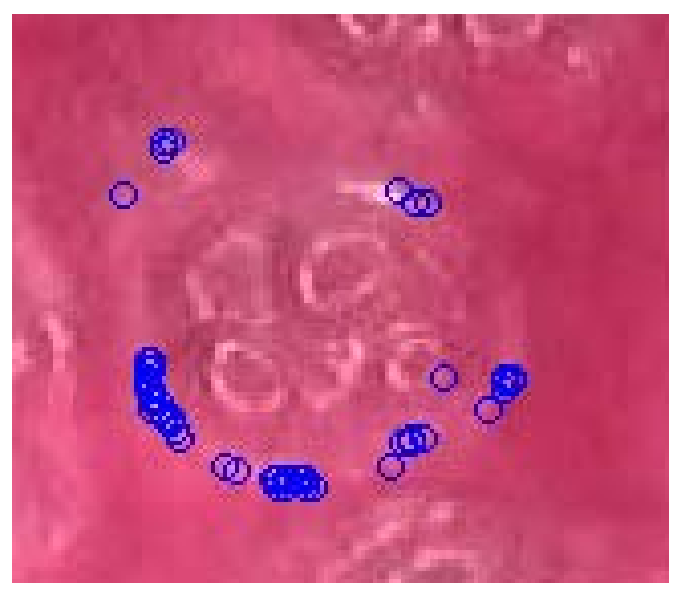

Figure 5. An example of thresholding the sum of the green and red channels to the locate edge points surrounding a type 3 alveolus group.

The next step of the method is to compute the convex hull of the group of points in Figure 5, which is essentially a piecewise linear approximation to the boundary of the alveolus group. The enclosed area is then computed which approximates the required area for this alveolus group and frame. An alternative approach to the linear approximation would be to use a cubic spline, but there are generally enough points so that there is negligible differences between these two approximations. The above method of Figures 4 and 5 is repeated for each subsequent frame in the image cycle.

\section{Alveoli tracking-final implementation}

In practice, more accurate tracking is achieved by starting at the peak frame or the end of inflation, and moving effectively "back in time" to the beginning of inflation. The algorithm then starts again at the peak frame, which is now the beginning of deflation and moves forward in time until the end 
of deflation. This approach minimizes the distance required to be tracked across the image improving the results, and is valid since this application does not require real-time tracking or feedback.

The end result of the tracking is that alveolus area data is found for the whole sequence of frames corresponding to a complete ventilator cycle from inflation to deflation. This data can be converted to an area versus time graph using the number of frames per second in the original video file.

Finally, the pressure versus time graph from the ventilator is synchronized with the video to create an overall pressure area curve for each group alveolus or single alveoli of interest. This curve is analogous to the quasi-static lung pressure/volume curve, except it depicts the change in individual alveolar area with increasing/decreasing airway pressure rather than the change in lung volume with increasing/decreasing airway pressure.

\section{E. Summary of automated alveoli tracking algorithm}

For clarity, the proposed alveoli tracking method is summarized in a flow chart as shown in Figure 6.

\section{RESULTS AND DISCUSSION}

The algorithm of Figure 6 is applied on the three types of alveoli, type 1 - healthy alveoli that has only a small change of area throughout inflation and deflation, type 2 - partially diseased alveoli that doesn't fully collapse at the end of deflation, type 3 - fully diseased alveoli that collapses at the end of deflation. These different types of alveoli are selected visually from the video and the corresponding regions of interest are used in steps 1 and 2 of the algorithm of Figure 6.

As a first test of robustness of the algorithm, several alveoli are tracked for multiple cycles. This test ensures that the algorithm satisfies the steady state behaviour of the alveoli. Figure 7 shows an example of tracking a group of type 3 alveoli over six cycles, with a period of approximately 1.5 seconds. All the cycles are very similar, validating the consistency of the method.

Note that since the contour surrounding the whole group of alveoli is tracked, there is extra area being added which is not part of any individual alveoli in the group. Therefore, the area at the end of each deflation period is larger than it should be. However, the overall shape and change in area will approximate the overall shape and change in area of the individual alveoli. Since this paper concentrates on the qualitative behaviour of the alveoli this error is not taken into account in the results but could be easily accounted for if required.

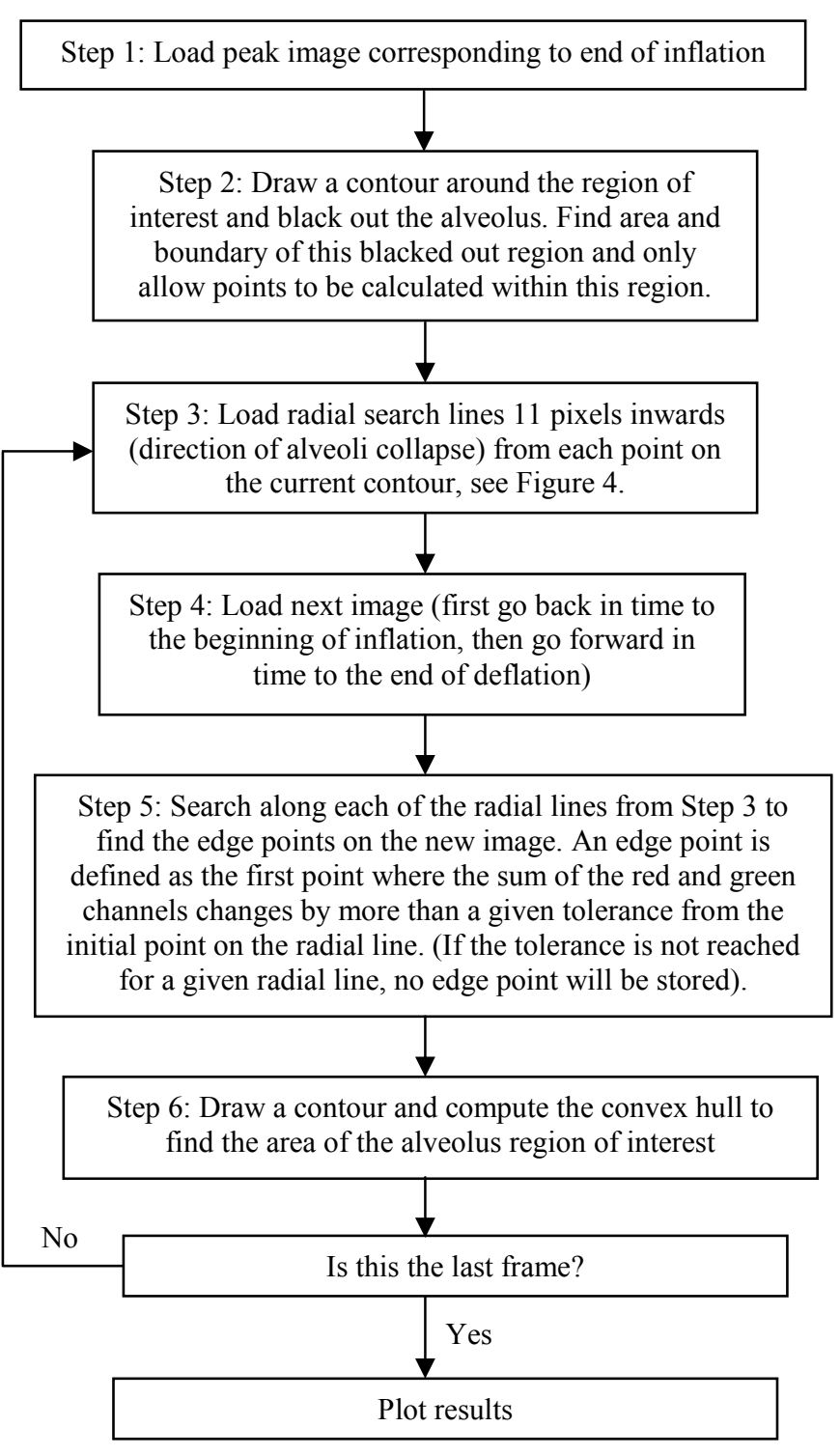

Figure 6. Summary of the proposed alveoli tracking method

\section{A. Type 1 alveoli}

A type 1 alveoli is healthy, so physiologically, it is not expected to change it's volume or area significantly throughout the ventilation cycle. To test this a single type 1 alveoli is tracked for approximately 7 seconds to ensure consistency. Figure 8 plots the result, where the red squares are the actual areas calculated by the algorithm and the dashed line is a linear interpolation.

The blue solid line is a smoothed version of the curve, which is constructed using an in-built cubic spline smoother function in Matlab. This blue line is likely a more realistic representation due to the image noise present. However, the 
raw data gives only a maximum deviation of $11 \%$ from the mean which is approximately 642 pixels. The smoothed curve on the other hand, which is more realistic, gives only a $4 \%$ change from the mean. These results show that there is very minimal change in the volume or area of this alveoli, which matches physiological expectation, and provides a further validation of the method.

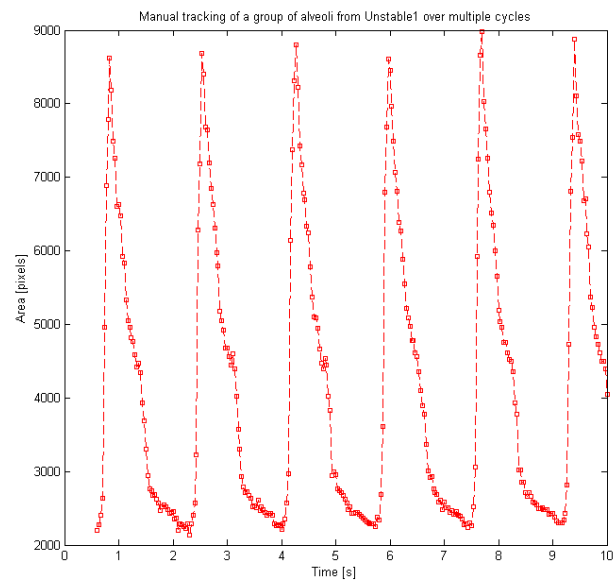

Figure 7. Tracking a group of type 3 alveoli over multiple cycles

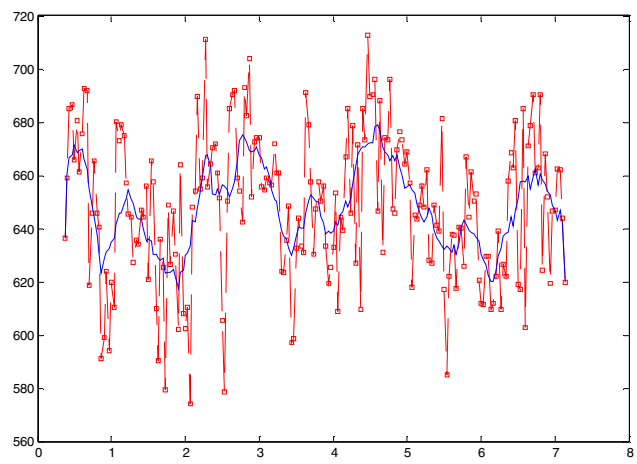

Figure 8. Tracking a single type 1 alveoli over multiple cycles

\section{B. Type 2 alveoli}

A type 2 alveoli is characterized by an increase in volume during inflation and a significant decrease in volume after deflation, but there is always some volume maintained at the lowest pressure. Figure 9 shows the area versus time and pressure for the tracking of a group of type 2 alveoli. Note that the normalized area is calculated by dividing the total area by the number of alveoli in the group and is measured in pixels, which is proportional to the physical alveoli area. Notice that in inflation, the largest change in volume occurs from 15-20 $\mathrm{mmHg}$ which shows that a type 2 alveoli pops open quite quickly.
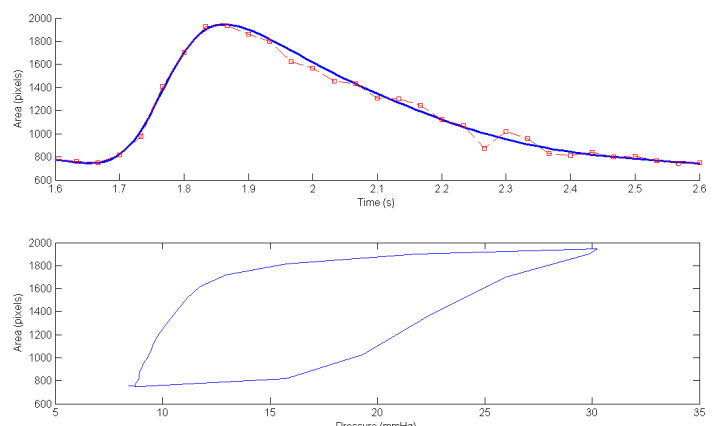

Figure 9. Area (pixels) versus time (s) and Area (pixels) versus pressure $(\mathrm{mmHg})$ respectively for a group of tracked type 2 alveoli

\section{Type 3 alveoli}

A type 3 alveoli has typically a greater increase in volume than a type 2 but can collapse at the end of deflation. Figure 10 shows the area versus time and pressure for the tracking of a group of type 3 alveoli. In this case, the biggest change in volume occurs from $23-27 \mathrm{mmHg}$ which shows that a type 3 alveoli pops open much later than a type 2. This result is consistent with the known physiological effect of greater stiffness in diseased alveoli, which requires more pressure to open.
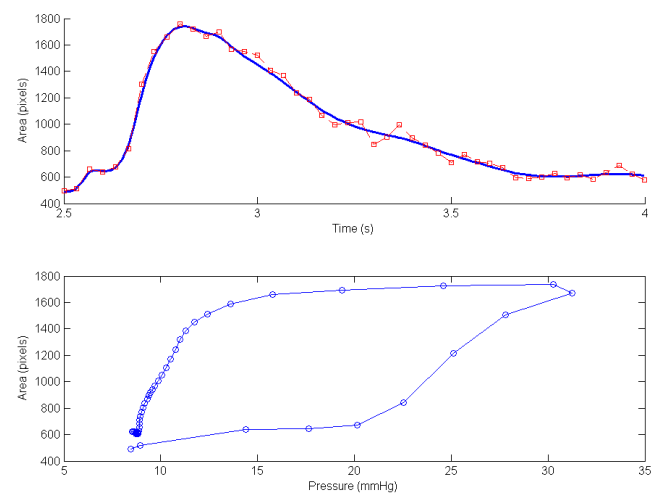

Figure 10. Area (pixels) versus time (s) and Area (pixels) versus pressure $(\mathrm{mmHg})$ respectively for a group of tracked type 2 alveoli

\section{Qualitative comparison with Schiller et al}

As a final validation, the pressure area curves of Figures 8-10 which correspond to the dynamic case of ventilation, are compared to the quasi-static derived pressure area curves in Schiller et al [4]. Note that they will not be the same quantitatively as different type 2 and 3 alveoli are being compared and there are significant transient effects in the dynamic case. The top plot in Figure 11 shows the results of 
Schiller et al. The bottom plot of Figure 11 consists of the inflation portions of Figures 9 and 10 scaled to approximately the same magnitude as Schiller et al in the top plot. This scaling may not be completely correct, as the calibration of the cameras in [4] was not available, but it allows a better comparison visually, and will not affect the overall shape. As can be seen in Figure 11, the individual curves in the bottom plot follow a similar trend to the curves in the top plot.

However, there are some important points of difference. For the type 2 alveoli, the first significant change in volume occurs from 15-20 $\mathrm{mmHg}$ for the dynamic case (tracking method), compared to $9-14 \mathrm{mmHg}$ in the quasi-static case (Schiller). For the type 3 alveoli, the first significant change of volume occurs from $17-24 \mathrm{mmHg}$ for the dynamic case compared to $\sim 22-25 \mathrm{mmHg}$ in the quasi-static case.

These results show that qualitatively, the alveoli Area pressure curves shift to the right when going from quasi-static ventilation to dynamic ventilation. This observation is consistent with the well known fact that in the quasi-static case, the pressure initially goes much higher before it decreases to a steady state value, for a given tidal volume increment. In other words, in the dynamic case, no time is given for the pressure to decrease, so for a given volume, all the pressures will be higher, resulting in a shift to the right.
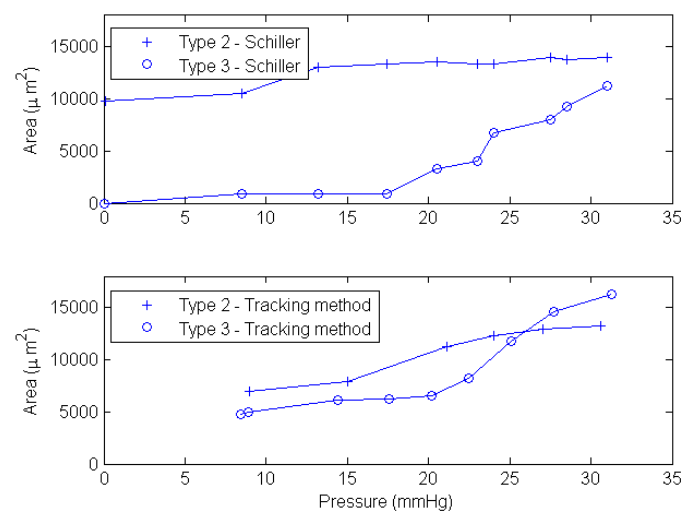

Figure 11. Area (pixels) versus time (s) and Area (pixels) versus pressure $(\mathrm{mmHg})$ respectively for a group of tracked type 2 alveoli

\section{CONCLUSION}

An alveoli tracking method was developed that was used to derive Area pressure curves for three different types of alveoli. The method tracks an initialized contour automatically for any specified number of ventilation cycles and gives reliable results in all cases. The algorithm utilizes a threshold on the change in the sum of the red and green channels to detect the boundaries of a single or group of alveoli. No image gradients are required so the method is robust to image noise and doesn't require significant tuning or filtering. Known information on the direction of expansion or contraction of the alveoli was an important feature in the success of the method.

The resulting tracking compared well visually with the movies, and the analyzed behaviour of each alveoli type was consistent with known physiological responses to ventilation. The results compared well qualitatively to existing results in the literature which rely on manual extraction of alveoli and static images.

In summary, the image-based alveoli tracking method can derive area pressure curves for healthy or diseased alveoli with minimal user intervention. This method allows the development of stress strain laws for different types of alveoli and will potentially lead to improved lung models and better management of acute lung injury in critical care patients.

\section{REFERENCES}

[1] A. Esteban, A. Anzueto, F. Frutos, et al, "Characteristics and outcomes in adult patients receiving mechanical ventilation: a 28-day international study" Jama, vol. 287(3), pp. 345-355, 2002.

[2] T Yuta. Minimal Model of Lung Mechanics for Optimising Ventilator Therapy in Critical Care, PhD Thesis, University of Canterbury, June 2007.

[3] D. Chiumello, E. Carlesso, P. Cadringher et al, "Lung Stress and Strain during Mechanical Ventilation for Acute Respiratory Distress Syndrome" Am J Respir Crit Care Med. ama, vol. 178, pp. 346-355, 2008

[4] H. J. Schiller, J. Steinberg, J. Halter et al, "Alveolar inflation during generation of a quasi-static pressure/volume curve in the acutely injured lung" Crit Care Med, vol. 31(4), pp. 1126-1133, 2003.

[5] C. Y. Xu, J. L. Prince, "Snakes, shapes, and gradient vector flow" IEEE Trans Image Processing, Vol 7: pp. 359-369, 1998.

[6] J. F. A. Canny, "Computational approach to edge detection" IEEE Trans Pattern Analysis and Machine Intelligence, vol. 8: pp. 679-698, 1986.

[7] D. G. Lowe, "Object recognition from local scale-invariant features" Proc IEEE 7th International Conference on Computer Vision, pp. 11501157.

[8] R. C. Hardie, and C. G. Boncelet, "Gradient-based edge detection using non-linear edge enhancing prefilters" IEEE Transactions on Image Processing, vol. 4(1): pp. 1572-1577, 1995. 